\title{
Diffusive-light invisibility cloak for transient illumination
}

\author{
B. Orazbayev, ${ }^{1}$ M. Beruete, ${ }^{1,2}$ A. Martínez, ${ }^{3}$ and C. García-Meca ${ }^{3, *}$ \\ ${ }^{1}$ Antennas Group-TERALAB, Universidad Pública de Navarra, Campus Arrosadía, 31006 Pamplona, Spain \\ ${ }^{2}$ Institute of Smart Cities, Public University of Navarra, 31006 Pamplona, Spain \\ ${ }^{3}$ Nanophotonics Technology Center, Universitat Politècnica de València, 46022 Valencia, Spain
}

(Received 2 September 2016; published 22 December 2016)

\begin{abstract}
Invisibility in a diffusive-light-scattering medium has been recently demonstrated by employing a scatteringcancellation core-shell cloak. Unlike nondiffusive cloaks, such a device can be simultaneously macroscopic, broadband, passive, polarization independent, and omnidirectional. Unfortunately, it has been verified that this cloak, as well as more sophisticated ones based on transformation optics, fail under pulsed illumination, invalidating their use for a variety of applications. Here, we introduce a different approach based on unimodular transformations that enables the construction of unidirectional diffusive-light cloaks exhibiting a perfect invisibility effect, even under transient conditions. Moreover, we demonstrate that a polygonal cloak can extend this functionality to multiple directions with a nearly ideal behavior, while preserving all other features. We propose and numerically verify a simple cloak realization based on a layered stack of two isotropic materials. The studied devices have several applications not addressable by any of the other cloaks proposed to date, including shielding from pulse-based detection techniques, cloaking undesired scattering elements in time-of-flight imaging or high-speed communication systems for diffusive environments, and building extreme optical security features. The discussed cloaking strategy could also be applied to simplify the implementation of thermal cloaks.
\end{abstract}

DOI: 10.1103/PhysRevA.94.063850

\section{INTRODUCTION}

For centuries, the ability to hide an object from the human eye has been one of the most fascinating products of mankind's imagination. However, it remained as a pure fictional idea until the rapid progress in electromagnetics occurred during the last decade, favored to a large extent by the rise of artificial materials, generically called metamaterials. Metamaterials have opened new possibilities for controlling electromagnetic fields, since their constitutive electromagnetic parameters can be tailored at will [1]. This inspired the creation of new powerful tools such as transformation optics (TO) $[2,3]$, where a space transformation is effectively induced by a suitable medium. As a result, a variety of cloaking mechanisms has been proposed [2-4] and even experimentally demonstrated [5,6]. In TO-based invisibility cloaks [2,3], the concealed object is isolated by redirecting the impinging wave around it, preventing any light absorption or scattering. Since this is usually achieved by means of an anisotropic and inhomogeneous medium with extreme values of the constitutive parameters, such cloaks are often highly complex and difficult to realize. Another important cloaking technique is scattering cancellation $[7,8]$, where the cloaking effect is achieved by suppressing the scattering harmonics from the concealed object [9]. Although this technique provides a simple and robust cloaking for subwavelength objects, the complexity of the design drastically increases with the size of the cloaked object. More recently, it has been demonstrated that an ultrathin metasurface, a simplified two-dimensional (2D) case of metamaterials, can provide a good cloaking performance for big objects on a ground plane with relatively wide bandwidth and angle span [10,11], facilitating the fabrication of the cloak.

\footnotetext{
*cargarm2@ntc.upv.es
}

Nevertheless, the TO and scattering cancellation principles are more appropriate for cloaking objects in the absence of a ground plane, and have been successfully adopted in different fields besides wave optics. For example, transformational cloaks have been designed for thermodynamics [12], acoustics [13], matter waves [14], and even for the space-time domain [15-17] and moving media [18,19]. Likewise, scattering cancellation has been applied, e.g., to the fields of light diffusion [20], elasticity, and acoustics [21,22].

In a diffusive medium, such as an organic tissue, milk, or dense fog, propagating photons undergo multiple collisions with the particles of the medium. This kind of propagation is described by the diffusion equation, allowing the application of the traditional scattering cancellation and TO cloaking principles in the static regime $[20,23]$. However, as it was demonstrated in Ref. [23], the diffusive-light cloak fails to conceal an object under truly transient conditions, whether it is based on a core-shell geometry or on typical coordinate transformations. In the first case, the disruption of the cloaking performance for transient illumination is attributed to the high diffusivity of the shell, which results in a faster light propagation in it than in the surrounding medium [23]. Thus, when the core-shell cloak is applied, a short illumination pulse will appear earlier for an external observer than in a medium with no object, with a subsequent fast drop of the intensity, making the cloaked object detectable. In turn, the TO-based cloak also fails, since, in diffusive-light processes, the timedependent diffusion equation is generally not form invariant under coordinate transformations (a basic requirement for this technique to work). In particular, the typical transformations that blow up a point to open a hole in space and achieve the cloaking effect change the form of this equation [23]. As a consequence, the TO method can only be partially implemented in this case, leading to a similar performance to that of a core shell, again rendering the cloak detectable in the transient regime. 
In this work, we show that there is a special type of coordinate transformations, namely, unimodular (compressionfree) transformations, which preserve the form of the timedependent diffusion equation. In addition, we propose a particular unimodular transformation able to reroute the flux of photons in a specific direction around a certain region, achieving a perfect unidirectional invisibility effect under truly transient conditions. Moreover, we demonstrate that an approximate multidirectional transient cloaking performance can be attained by applying (polygonal) linear coordinate transformations [24]. The proposed cloak has an additional advantage, as it only requires a homogeneous anisotropic medium for its implementation. We demonstrate numerically that such a medium can be replaced by a thin layered system of two isotropic materials, facilitating the practical realization of the cloak.

\section{UNIDIRECTIONAL CLOAK}

Using Fick's first law, the generic time-dependent diffusion equation reads [20]

$$
\nabla \cdot\left(D_{0} \nabla \phi\right)-C_{0} \frac{\partial \phi}{\partial t}=0
$$

where $t$ denotes time and $\phi$ denotes some diffusing quantity (possible physical meanings for the coefficients $D_{0}$ and $C_{0}$ will be discussed next). After applying a coordinate transformation (with the aim of molding the diffusion flux, see below), the previous equation becomes [12,23]

$$
\nabla^{\prime} \cdot\left(\Lambda D_{0} \Lambda^{\top} \frac{1}{\operatorname{det} \Lambda} \nabla^{\prime} \phi\right)-\frac{C_{0}}{\operatorname{det} \Lambda} \frac{\partial \phi}{\partial t}=0,
$$

where $\nabla^{\prime}$ is the gradient with respect to the new coordinates, $\Lambda$ is the Jacobian matrix of the coordinate transformation, and $\Lambda^{\top}$ is its transpose. As it can be seen, the coefficient associated with the time derivative in Eq. (2) is different from unity. In the case of thermodynamics, $\phi$ represents the temperature $T$, $D_{0}$ is the thermal conductivity tensor, and $C_{0}$ is the specific heat. Hence, Eq. (2) can be interpreted as that corresponding to a second medium characterized by a new set of parameters:

$$
\begin{gathered}
D=\frac{\Lambda D_{0} \Lambda^{\top}}{\operatorname{det} \Lambda}, \\
C=\frac{C_{0}}{\operatorname{det} \Lambda},
\end{gathered}
$$

which induces the desired transformation [12]. However, in the case of light diffusion, for which $\phi$ represents the photon density $n_{\mathrm{P}}$ and $D_{0}$ is the diffusivity, the coefficient of the time derivative $\left(C_{0}\right)$ is always equal to 1 (since there is only one material parameter for the diffusion process - the diffusivity $D_{0}$ ). Therefore, the condition specified by Eq. (4) cannot be fulfilled in general. This is not important in the static (or quasistatic) regime, in which the time derivative term is negligible and the diffusivity is the only relevant parameter. However, this is no longer true in the transient regime, in which using a material with a diffusivity given by Eq. (3) will not be enough to mimic the transformed equation. This results in the imperfect invisibility performance discussed in Ref. [23].

However, one can see from Eq. (2) that, for a transformation with $\operatorname{det} \Lambda=1$, the transformed light diffusion equation preserves its original form. This means that the Jacobian matrix has to be unimodular (note that transformations with $\operatorname{det} \Lambda=-1$ are also unimodular, although we will not consider them in this work), which requires a coordinate transformation without compression of space. In this case, the transformed equation can be exactly implemented only with a diffusivity given by Eq. (3). This fact has been overlooked so far.

Although the no-compression condition limits the achievable functionalities, it is still possible to obtain a cloaking effect in specific situations. As an example, we propose here a compression-free mapping that yields unidirectional invisibility under transient conditions. Figure 1(a) shows how this transformation maps a rectangular grid in the original space, described by a Cartesian coordinate system $(x, y, z)$, to physical space, described by a coordinate system $\left(x^{\prime}, y^{\prime}, z^{\prime}\right)$.

Specifically, an affine mapping is applied to the region $-c<x<c,-a<y<a$, while the identity transformation is applied outside the transformed counterpart of this region. For the first quadrant $(0<x<c, 0<y<a)$, the transformation equations can be written as

$$
x^{\prime}=x, \quad y^{\prime}=y+k(c-x), \quad z^{\prime}=z,
$$

with symmetric expressions for the other quadrants. Note that this transformation is unimodular everywhere.

As can be seen, the segment $(y=0,-c<x<c)$ is blown up to a rhombic region with side length $b$ and angle $\alpha$ ( $k=\tan \alpha$ and $c=b / \cos \alpha$ ), which will constitute the cloaked region.

Using Eq. (3) and taking advantage of the symmetries of the structure, the diffusivity tensor is obtained for the first and third ( - sign $)$ and second and forth $(+$ sign) quadrants as

$$
D=D_{0}=\left(\begin{array}{ccc}
1 & \mp k & 0 \\
\mp k & 1+k^{2} & 0 \\
0 & 0 & 1
\end{array}\right)
$$

where $D_{0}$ is the diffusivity of the surrounding medium. Clearly, Eq. (6) represents a homogeneous anisotropic medium.

From the schematic illustration in Fig. 1(a), it can be seen that the diffusive-light flux is redirected by the transformed medium (blue) around the cloaked region without compressing space. Note that, in order to efficiently conceal an object, the aperture $a$ of the cloak has to be larger than the incident beam waist, since the transformation is not continuous at the upper and lower cloak boundaries. In addition, it is worth mentioning that a double carpet cloak transformation as the ones employed in Refs. [25,26] would not satisfy the unimodular condition, since its determinant is constant but different from unity.

To verify the proposed design, numerical simulations were performed using COMSOL Multiphysics. In all simulations the diffusivity of the background medium was fixed to $D_{0}=$ $7.5810^{4} \mathrm{~m}^{2} / \mathrm{s}$ (as in Ref. [23]), while losses were neglected for the sake of simplicity. The structures were illuminated by a Gaussian-shape beam with a diameter of $2 w_{0}=64 \mathrm{~mm}$. A cloak with an aperture $2 a=70 \mathrm{~mm}$ was used to conceal a diffusively isolated (with $-\mathbf{n} \cdot \mathbf{j}=0, \mathbf{n}$ being the unit vector normal to the boundary of the cloaked region and $\mathbf{j}=D \nabla n_{\mathrm{P}}$ the photon flux) square object $\left(\alpha=45^{\circ}\right)$ with side $b=15 \mathrm{~mm}$ [Fig. 1(a)]. Note that the aperture of the cloak was chosen to be close to the incident beam waist, in order to account for the effect of the beam size on the cloak behavior. 

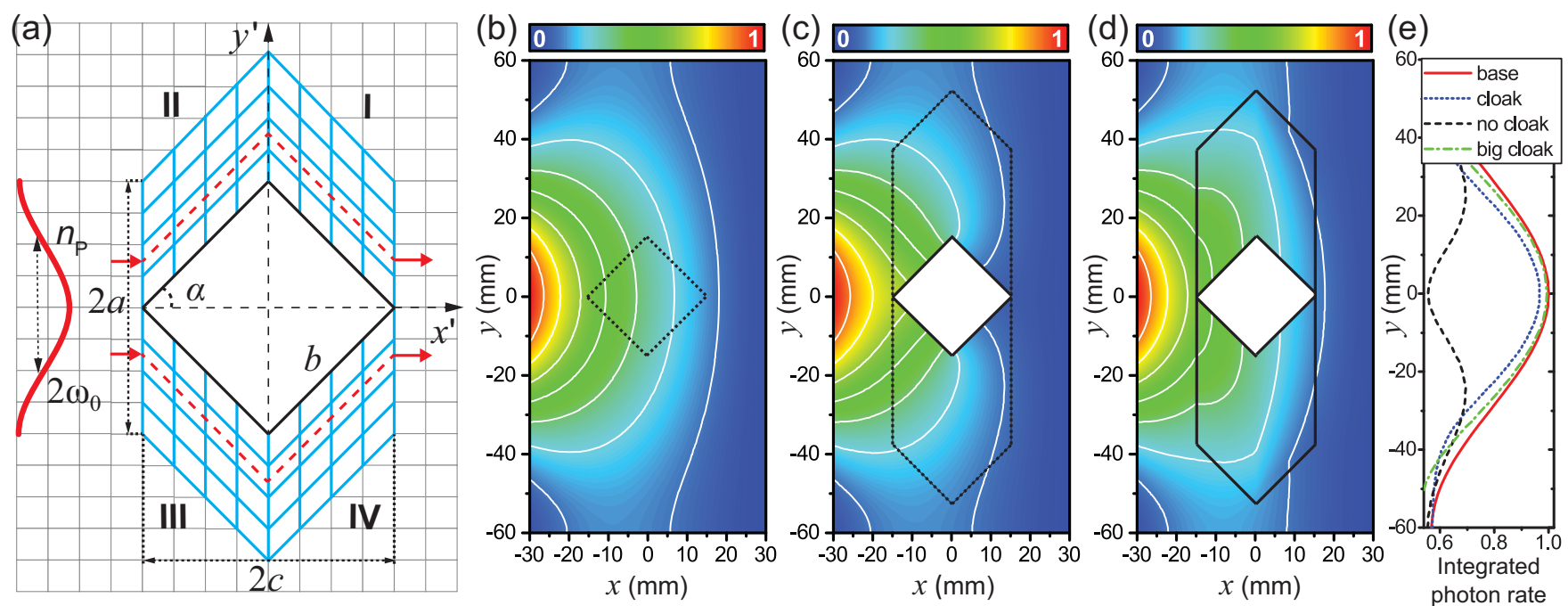

FIG. 1. (a) Transformation employed to build the proposed TO-based diffusive-light cloak. (b-d) Normalized numerical photon density distribution for the cases (b) no object, (c) only object, and (d) object with cloak. (e) Transmitted photon rate integrated over time at $x=30 \mathrm{~mm}$ for no object (solid red line), only object (dashed black line), object with cloak (dotted blue line), and object with cloak of aperture $2 a=90 \mathrm{~mm}$ (dash-dotted green line). The incident photon flux has a Gaussian shape with radius $w_{0}=32 \mathrm{~mm}$.

First, the performance of the unidirectional cloak was checked in the static case $\left(\partial n_{\mathrm{P}} / \partial t=0\right)$. The numerical results for the normalized photon density distribution for the cases without object, with bare object, and with cloaked object are shown in Figs. 1(b)-1(d). From these figures it is obvious that, when the cloak is applied, the photon density distribution is restored to that in which no object is present. However, due to the similar size of the beam waist and the aperture of the cloak, the transmitted photon rate (photon flux going through the boundary at $x=30 \mathrm{~mm}$ ) integrated over time is slightly smaller than in the ideal case (-3\%), as seen in Fig. 1(e) (blue dotted line).

In order to quantify how well our cloak performs, we used the root-mean-square error (RMSE), defined as

$$
\text { RMSE }=\sqrt{\frac{1}{m} \sum_{i=1}^{m}\left[T_{\text {base }}\left(y_{i}\right)-T_{\text {cloak }}\left(y_{i}\right)\right]^{2}},
$$

where $T_{\text {base }}\left(y_{i}\right)$ and $T_{\text {cloak }}\left(y_{i}\right)$ are the normalized transmitted photon rate distributions at the point $y_{i}$ for the ideal case without object and for the cloaked object, respectively ( $m$ being the total number of samples in the $y$ direction). For the studied case, a corresponding low value of RMSE $=0.05$ is obtained, which can be further reduced by either increasing the cloak aperture or decreasing the incident beam waist. For example, for the same incident flux and a cloak aperture $2 a=$ $90 \mathrm{~mm}$, the transmitted photon rate distribution is almost equal to the ideal one, see Fig. 1(e) (green dash-dotted line), with an RMSE $=0.03$ (the RMSE tends to zero as we increase the cloak aperture). As a reference, a much higher RMSE $=0.22$ is obtained for the bare object case.

Next, we verified the performance of the cloak in the transient regime. In order to be a truly transient illumination source, its temporal variations have to be shorter than the propagation time in the medium. Therefore, the duration of the pulse is determined by a diffusion time constant, which depends on the diffusivity $D$ and the dimensions of the structure. For the chosen parameters, the diffusion time constant is around 5 ns. Thus, to ensure the transient illumination conditions, a pulse with both Gaussian temporal (duration of $500 \mathrm{ps,} \mathrm{similar} \mathrm{to} \mathrm{that} \mathrm{employed} \mathrm{in} \mathrm{Ref.} \mathrm{[23])}$ and Gaussian spatial $\left(w_{0}=32 \mathrm{~mm}\right)$ distribution was chosen as the source. The rest of the employed parameters were the same as in the static case. The numerical results for the time evolution of the spatial distribution of the transmitted photon rate (at $x=30 \mathrm{~mm}$ ) are shown in Figs. 2(a), 2(d), and 2(g) for the medium without object, with uncloaked object, and with cloaked object, respectively. As it can be seen, when the cloak is applied, the photon rate distribution is restored in space as well as in time. Note that, as in the static case, the mentioned shadow effect is present, resulting in a slightly smaller spot.

To explicitly demonstrate that this cloak works under transient illumination, we compare its performance with the cylindrical core-shell cloaks studied in Refs. [20,23]. For the core-shell simulations, we used the same background diffusion coefficient and dimensions as in the previous simulations. Since all media are assumed to be lossless, the diffusive coefficient of the shell was calculated using the formula derived in Ref. [20] for ideal cloaking. The ratio of the outer to inner radii of the cloak was fixed to $R_{2} / R_{1}=1.25$, giving a diffusivity coefficient for the shell of $D_{2}=3.58 \times$ $10^{4} \mathrm{~m}^{2} / \mathrm{s}$, where $R_{1}=16 \mathrm{~mm}$ and $R_{2}=20 \mathrm{~mm}$ are the inner and outer radii, respectively (same dimensions as in Ref. [23]). The illumination conditions and the rest of the simulation parameters were kept the same. The results, shown in Figs. 2(b), 2(e), and 2(h), are very similar to the numerical and experimental results obtained in Ref. [23], demonstrating that the core-shell geometry fails in the transient regime. In contrast, the proposed unidirectional cloak recovers almost perfectly the distribution of the original transmitted diffusive wave. Analogously to the static case, the performance of this cloak can be further improved by increasing its aperture. 


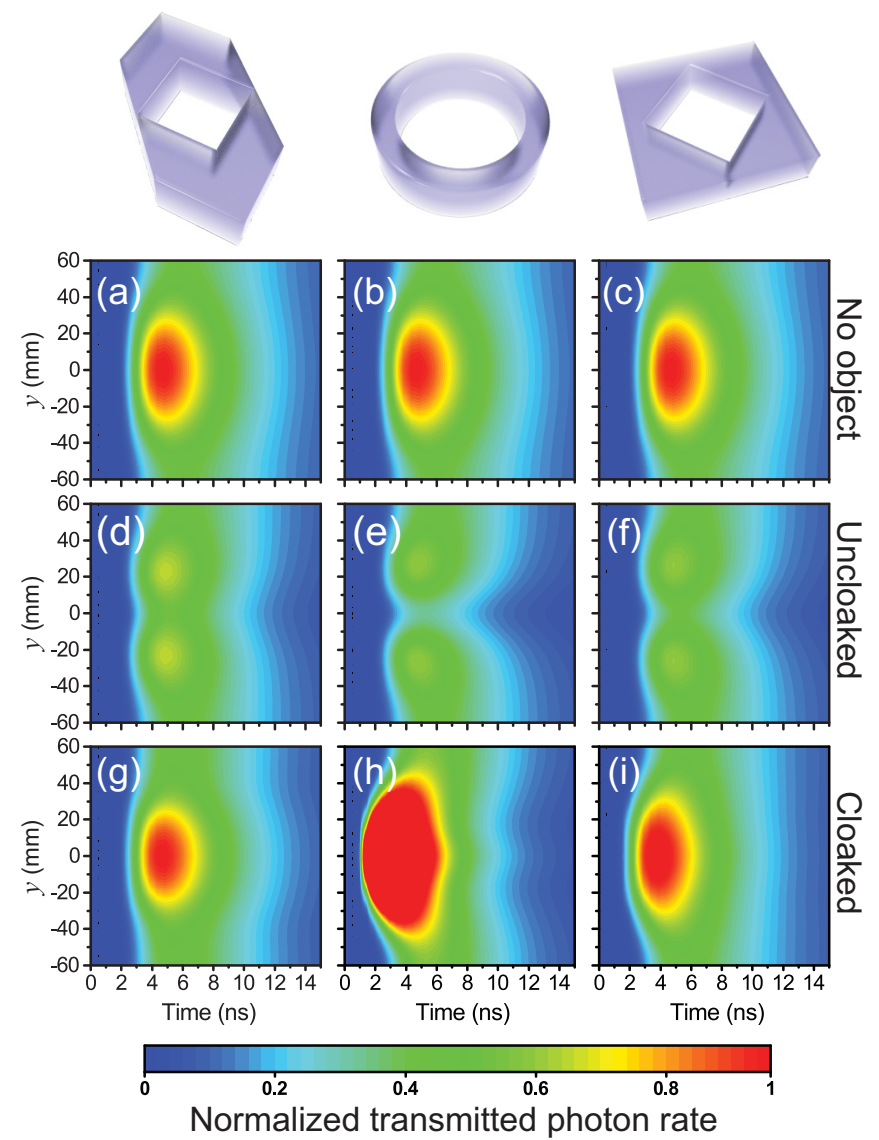

FIG. 2. Numerical results for the time evolution of the transmitted photon rate for the proposed unidirectional cloak (left column), the cylindrical core-shell cloak (center column), and the proposed polygonal cloak (right column). The photon rate distribution for the background medium (first row), uncloaked object (second row), and cloaked object (third row) is shown.

For completeness, we analyzed the performance of the proposed cloak at oblique incidence using the previous RMSE method. The calculated time evolution of the RMSE as a function of the incidence angle is shown in Fig. 3(a). Following this criterion and according to Fig. 3, the proposed diffusive cloak can still provide a good reconstruction (with a maximum RMSE $<0.09$, more than two times smaller than that for the bare object case, RMSE $=0.22$ ) of the transmitted photon rate distribution, i.e., hide the object, for small incidence angles (in an angular span of $\sim 8^{\circ}$ ), even though it was initially designed to work at $0^{\circ}$. Note that this range can be expanded by simply increasing the aperture of the cloak. For comparison purposes, the time evolution of the calculated RMSE for the core-shell cloak is shown in Fig. 3(b), which is insensitive to the incidence direction thanks to the symmetry of the structure. It can be clearly seen that this device fails in the transient regime, which manifests in the high values of RMSE $=0.45$ (even higher than the error for the case of the bare cylinder, $\operatorname{RMSE}=0.3$ ) around $t=2 \mathrm{~ns}$.

\section{MULTIDIRECTIONAL CLOAK}

The cloak simplifications here performed (limitation of the incident beam size and restriction to a specific propagation (a)
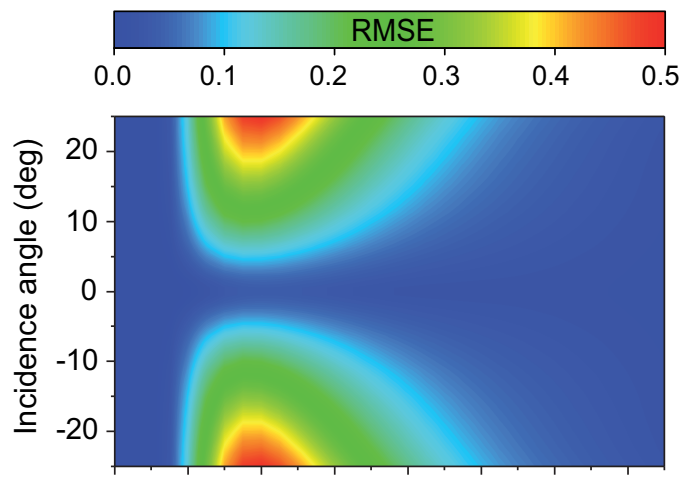

(b)

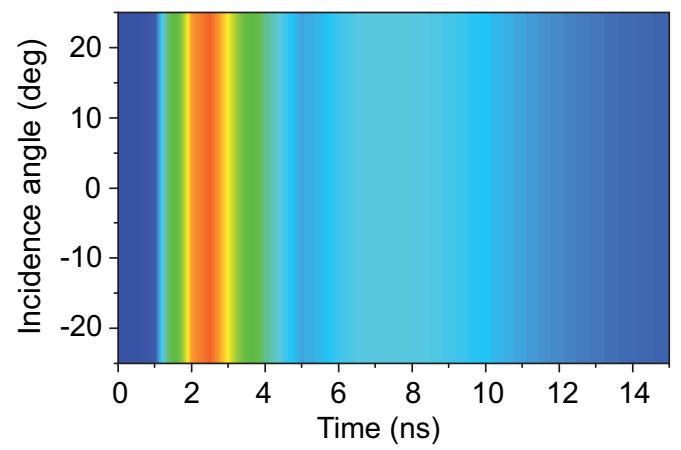

FIG. 3. Numerical results for the time evolution of the RMSE distribution vs incidence angle for (a) proposed unidirectional cloak and (b) cylindrical core-shell cloak.

direction) allowed us to apply the TO formalism not only for the static regime but also for the transient one. However, in some applications the direction and size of the incident beam may not be known from the onset, in which case a multidirectional cloak is desirable. In such a type of cloak, the creation of a hidden region requires the compression of space and, consequently, a transformation that does not preserve the form of the light diffusion equation. For example, a cylindrical (in 2D geometry) TO-based cloak [2], despite its highly complex and hard-to-build design (due to the spatial anisotropy and inhomogeneity of the diffusivity), has a similar performance to that of the core-shell cloak, as demonstrated in Ref. [20].

We wondered, however, whether the suppression of the inhomogeneity of the transformation could improve the performance of a multidirectional cloak under transient illumination. The reason is that, in this case, $\operatorname{det} \Lambda$ would be constant inside the cloak (although not equal to 1) and could be taken out of the spatial derivative in Eq. (2), canceling out the coefficient of the time derivative and yielding an effective coefficient $C=1$, as desired. Unfortunately, det $\Lambda=1$ outside the cloak. This discontinuity in det $\Lambda$ cannot be avoided (preventing us from taking it out from the spatial derivative) and is a possible source of degradation of the cloak performance in the transient regime. To analyze this possibility, we considered an $N$-sided polygonal cloak, which makes a large region appear as a much smaller one, rendering it virtually invisible [24]. Specifically, we studied a square cloak $(N=4)$ with side $a=45 \mathrm{~mm}$ [blue square in Fig. 4(a)]. To achieve the cloaking effect, the space between the blue square and a second small square of 

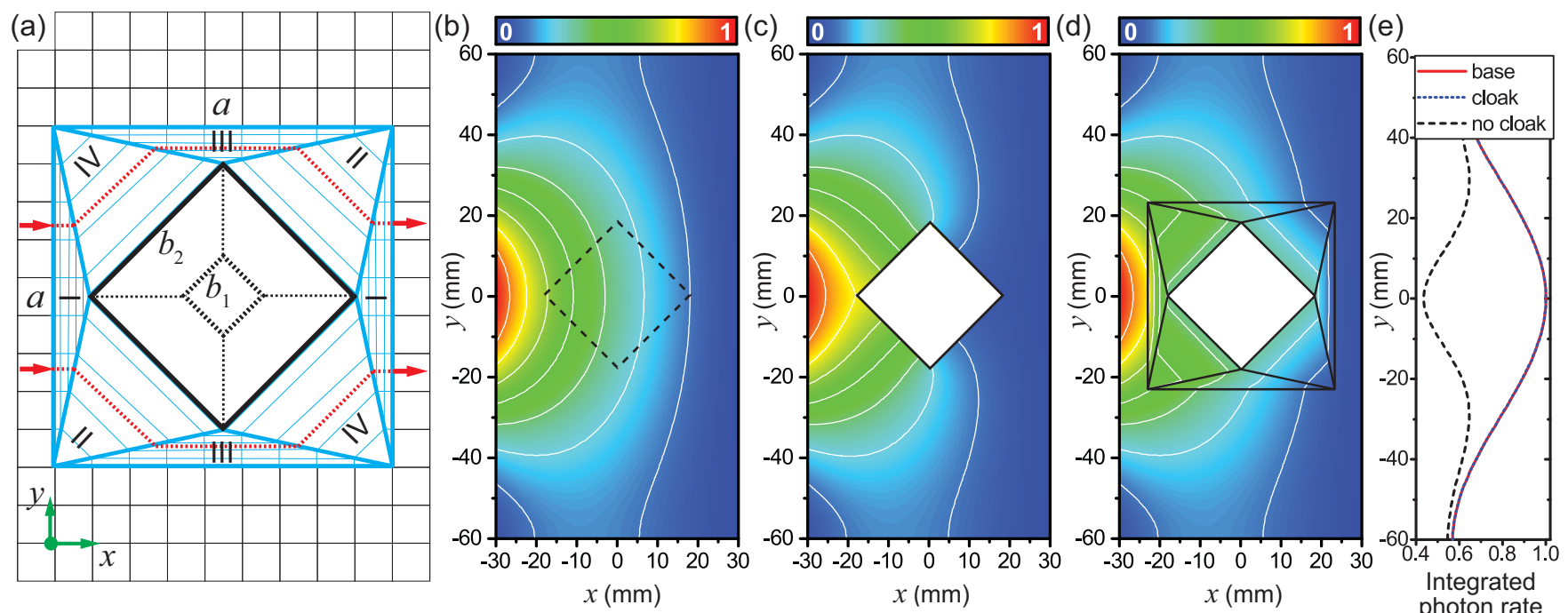

FIG. 4. (a) Scheme of the proposed multidirectional diffusive-light cloak. (b-d) Normalized numerical photon density distribution in the static regime for the cases (b) no object, (c) bare object, and (d) object with cloak. (e) Transmitted photon rate, integrated over time at $x=30 \mathrm{~mm}$, for no object (solid red line), only object (dashed black line), and object with cloak (dotted blue line).

negligible area [black dotted-line square in Fig. 4(a), with side $b_{1}=1 \mathrm{~mm}$ and rotated by an angle of $45^{\circ}$ with respect to the outer blue square] is divided into multiple regions. Finally, a linear coordinate transformation is performed in each region in order to expand the small square to a much larger one [black solid-line square in Fig. 4(a), with side $b_{2}=25 \mathrm{~mm}$ ], generating the cloaked region [24].

Taking advantage of the symmetries of the structure, only four different regions need to be considered, the corresponding transformation equations of which can be written as

$$
\begin{array}{lr}
\text { I : } & x^{\prime}=\frac{x}{k_{1}}, \quad y^{\prime}=y, \quad z^{\prime}=z, \\
\text { II : } \quad x^{\prime}=k_{2} x, \quad y^{\prime}=y-k_{2} x, & z^{\prime}=z, \\
\text { III : } \quad x^{\prime}=x, \quad y^{\prime}=\frac{y}{k_{1}}, & z^{\prime}=z, \\
\text { IV : } \quad x^{\prime}=k_{2} x, \quad y^{\prime}=y+k_{2} x, \quad z^{\prime}=z,
\end{array}
$$

where $k_{1}=\left(a-\sqrt{2} b_{1}\right) /\left(a-\sqrt{2} b_{2}\right)$ and $k_{2}=b_{2} / b_{1}$. This transformation gives rise to a homogeneous anisotropic transformed medium.

As in the previous cases, the performance of this device was analyzed through numerical simulations, considering that the cloaked region (delimited by the black solid-line square) is diffusively isolated. According to the results depicted in Figs. 4(b)-4(d), in the static regime, the distribution of the photon density outside the device remains unperturbed, confirming the ideal cloaking of the object. Figure 4(e) further demonstrates that the spatial distribution of the transmitted photon rate integrated over time in the case of the applied cloak (blue dotted line) is identical to that in which no object is present (red solid line).

Finally, we checked the performance of the polygonal cloak in the transient regime. As in the previous designs, the spatial distribution of the transmitted photon rate was obtained as a function of time for three different cases (no object, uncloaked object, and cloaked object). The results are shown in Figs. 2(c), 2(f), and 2(i). As expected, since the form of the diffusion equation cannot be completely preserved in this case, the cloaking effect is not perfect. However, it is much closer to the ideal one than that achieved via scattering cancellation. For instance, unlike in the case of the core-shell cloak, the polygonal cloak hardly causes an overshooting of intensity in the beginning (the spot occurs less than $1 \mathrm{~ns}$ earlier in time than in the reference case). In fact, the shape of the pulse is approximately the ideal one at all times, making the cloaked object much more difficult to detect.

\section{PRACTICAL MULTILAYER IMPLEMENTATION}

Unlike the cylindrical TO cloak, the diffusive medium required to synthesize the cloak proposed in Sec. II is homogeneously anisotropic. In this section, we show that this fact can greatly simplify its implementation. We start by noticing that, thanks to its real symmetric character, the diffusivity tensor given by Eq. (6) becomes diagonal when expressed in a Cartesian system the axes of which lie along its eigenvectors. This so-called principal coordinate system $(u, v, z)$ can be obtained as a rotation of the original coordinate system $(x, y, z)$ by an angle $\theta$ around the $z$ axis [Fig. 5(a)].

For the cloak parameters used in this work $(k=\tan \alpha=1)$, the principal components (eigenvalues) of the diffusivity tensor in the first quadrant are determined as $D^{u}=0.38 D_{0}$ and $D^{v}=2.62 D_{0}$, with a corresponding rotation angle of $\theta=31.72^{\circ}$. The diffusivity tensor principal components for the other quadrants can be easily obtained via symmetry considerations. From homogenization theory [27], we know that such an anisotropic medium can be replaced by a system of alternating thin layers of two different materials A and B $[25,28]$, aligned with the mentioned principal axes and with isotropic diffusivities $D_{\mathrm{A}}$ and $D_{\mathrm{B}}$ and thicknesses $t_{\mathrm{A}}$ and $t_{\mathrm{B}}$, respectively [see Fig. 5(a)].

Specifically, such a composite behaves as an effective anisotropic medium characterized by a diffusivity tensor with 

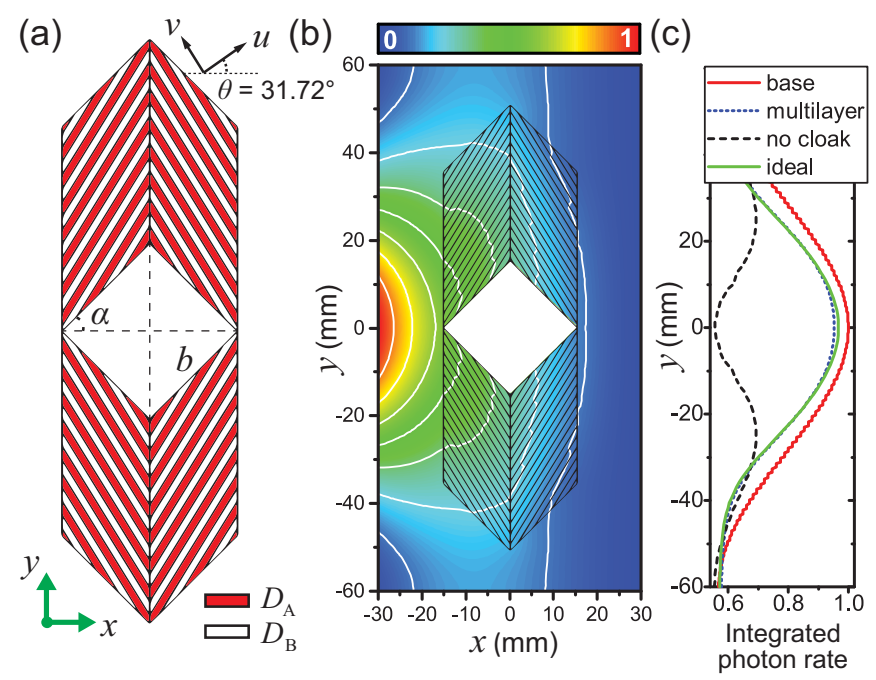

FIG. 5. (a) Scheme of the proposed multilayered diffusive-light cloak. $D_{\mathrm{A}}=38.2 \times 10^{4} \mathrm{~m}^{2} / \mathrm{s}\left(5 D_{0}\right), D_{\mathrm{B}}=1.5 \times 10^{4} \mathrm{~m}^{2} / \mathrm{s}\left(0.2 D_{0}\right)$. (b) Normalized photon density distribution in the presence of the cloaked object. (c) Transmitted photon rate at $x=30 \mathrm{~mm}$ integrated over time for no object (solid red line), only object (dashed black line), object with multilayered cloak (dotted blue line), and object with ideal cloak (dash-dotted green line). The incident photon flux has a Gaussian shape with radius $w_{0}=32 \mathrm{~mm}$.

principal components given by

$$
D^{u}=\frac{(1+f) D_{\mathrm{A}} D_{\mathrm{B}}}{f D_{\mathrm{A}}+D_{\mathrm{B}}}, \quad D^{v}=\frac{D_{\mathrm{A}}+f D_{\mathrm{B}}}{1+f},
$$

where $f=t_{\mathrm{B}} / t_{\mathrm{A}}$. For simplicity, we assumed here layers of equal thickness $t_{\mathrm{A}}=t_{\mathrm{B}}=t$ (that is, $f=1$ ). It should be noted that an adaptive step discretization can provide a better performance in typical cloaks where the material parameters are spatially varying [29]. However, due to the homogeneity of the proposed cloaking diffusive medium, the use of an equal-step discretization is a suitable choice in our case. Using Eq. (9), we can calculate the values of the multilayer isotropic diffusivities that implement the anisotropic medium required by the cloak, which were found to be $D_{\mathrm{A}}=5 D_{0}=$ $38.2 \times 10^{4} \mathrm{~m}^{2} / \mathrm{s}$ and $D_{\mathrm{B}}=0.2 D_{0}=1.5 \times 10^{4} \mathrm{~m}^{2} / \mathrm{s}$. Note that the thickness $t$ of the layers has to be small enough to have a correct effective medium diffusivity. In this example, we used $t=2 \mathrm{~mm}$. In order to verify that this value fulfills the mentioned requirement, the behavior of the multilayered diffusive cloak was numerically analyzed as well.

Analogously to the ideal homogeneous anisotropic cloak, Fig. 5(b) shows that the multilayered cloak restores the photon density distribution to the case in which no object is present. Furthermore, the spatial distribution of the integrated-overtime transmitted photon rate in the case of the multilayered cloak (blue dotted line) is almost identical to the distribution obtained when the ideal homogeneous cloak is applied (green dash-dotted line) [see Fig. 5(c)]. Moreover, in the transient regime, the spatial distribution of the transmitted photon rate evolves in time identically to that obtained for the ideal cloak (see Fig. 6), proving the validity of the proposed structure.

This multilayered system significantly facilitates the practical realization of the cloak, which becomes technologically

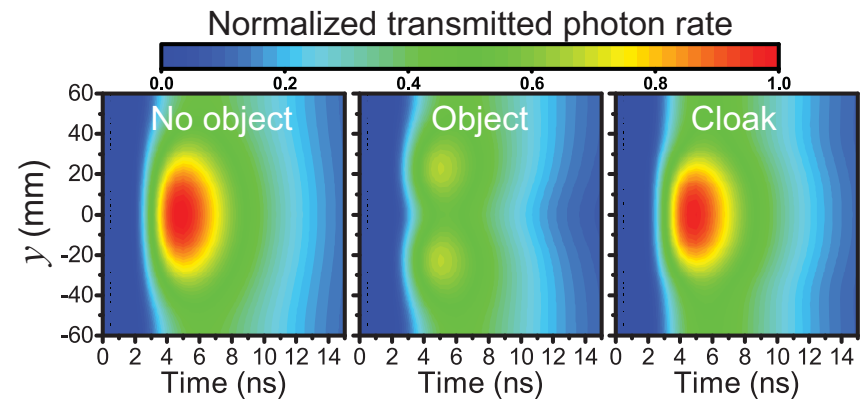

FIG. 6. Numerical results for the time evolution of the transmitted photon rate for the proposed multilayered unidirectional cloak. The photon rate distribution for the background medium (first column), uncloaked object (second column), and cloaked object (third column) is shown.

comparable to that of the core-shell cloaks based on scattering cancellation. For instance, the required thin layers with different diffusive coefficients $D_{\mathrm{A}}$ and $D_{\mathrm{B}}$ could be fabricated using solid-state materials (for example, a transparent polymer polydimethylsiloxane doped with titania nanoparticles), as it was shown in Ref. [30], and attached altogether afterwards. Note that similar layered systems could be employed to implement the studied polygonal cloak.

\section{CONCLUDING REMARKS}

In this work, we have proposed and numerically analyzed a unidirectional diffusive-light cloak based on TO, which can provide an almost perfect invisibility effect in both the static and transient regimes. Additionally, we have demonstrated that it is possible to obtain a multidirectional cloaking displaying a nearly ideal performance in the transient regime by using a polygonal cloak. It is worth mentioning that this last technique could even be extended to the three-dimensional case [31].

From the fabrication standpoint, we have proposed a simple multilayered implementation of the studied cloak that facilitates its practical realization. Such a thin layered system could be fabricated using solid-state diffusive materials.

Our results have several potential applications that cannot be addressed by the diffusive cloaks previously proposed (for instance, those in Refs. [20,23]). First, the cloaks studied here offer a complete shielding strategy for advanced pulse-based detection techniques such as LIDAR. Second, this kind of cloak could be used to avoid interferences from scattering elements for high-speed communications in a diffusive environment. As a third example, in previous works it has been mentioned that any diffusive cloak could be detected by time-of-flight tomography. Although this seems to be true in the ideal case in which any number of directions can be analyzed in a very precise way, the cloaks that we have proposed in this paper could be very difficult to detect if only a limited (arbitrary, in principle) number of directions is considered, which may be the case in some real systems. This feature could be used to hide certain elements of an imaging system susceptible to introducing interferences, or to build even more sophisticated optical security elements [32] than the ones based on static diffusive cloaks [23]. 
Moreover, the cloaking strategy here discussed could also be applied in the field of thermodynamics. Even though the heat equation is form invariant under general spatial coordinate transformations, a full implementation requires an independent control over the medium thermal conductivity and specific heat, which is challenging in practice [33]. Conversely, the thermal analogs of the considered diffusive cloaks would only demand an engineered diffusivity. It is worth mentioning that there exist alternative cloaking techniques that can also relax the implementation complexity of thermal cloaks [34,35], as well as of dc current cloaks [36].

\section{ACKNOWLEDGMENTS}

This work was supported in part by the Spanish Ministerio de Economía y Competitividad under Contracts No. TEC201451902-C2-1-R and No. TEC2014- 51902-C2-2-R. B.O. is sponsored by the Spanish Ministerio de Economía y Competitividad under Grant No. FPI BES-2012-054909. M.B. is sponsored by the Spanish Ministerio de Economía y Competitividad via Grant No. RYC-2011-08221. C.G.-M. acknowledges support from Generalitat Valenciana through the VALi+d postdoctoral program (Grant No. APOSTD/2014/044).
[1] R. Marqués, F. Martín, and M. Sorolla, Metamaterials with Negative Parameters: Theory, Design, and Microwave Applications (Wiley, New York, 2008).

[2] J. B. Pendry, D. Schurig, and D. R. Smith, Science 312, 1780 (2006).

[3] U. Leonhardt, Science 312, 1777 (2006).

[4] J. Li and J. B. Pendry, Phys. Rev. Lett. 101, 203901 (2008).

[5] D. Schurig, J. J. Mock, B. J. Justice, S. A. Cummer, J. B. Pendry, A. F. Starr, and D. R. Smith, Science 314, 977 (2006).

[6] M. Gharghi, C. Gladden, T. Zentgraf, Y. Liu, X. Yin, J. Valentine, and X. Zhang, Nano Lett. 11, 2825 (2011).

[7] A. Alù and N. Engheta, Phys. Rev. E 72, 016623 (2005).

[8] B. Edwards, A. Alù, M. E. Young, M. Silveirinha, and N. Engheta, Phys. Rev. Lett. 100, 033903 (2008).

[9] F. Monticone and A. Alù, Phys. Rev. X 3, 041005 (2013).

[10] N. Mohammadi Estakhri and A. Alù, IEEE Antennas Wirel. Propag. Lett. 13, 1775 (2014).

[11] B. Orazbayev, N. Mohammadi Estakhri, M. Beruete, and A. Alù, Phys. Rev. B 91, 195444 (2015).

[12] S. Guenneau, C. Amra, and D. Veynante, Opt. Express 20, 8207 (2012).

[13] H. Chen and C. T. Chan, J. Phys. D. Appl. Phys. 43, 113001 (2010).

[14] S. Zhang, D. A. Genov, C. Sun, and X. Zhang, Phys. Rev. Lett. 100, 123002 (2008).

[15] M. W. McCall, A. Favaro, P. Kinsler, and A. Boardman, J. Opt. 13, 024003 (2011).

[16] C. García-Meca, S. Carloni, C. Barceló, G. Jannes, J. SánchezDehesa, and A. Martínez, Wave Motion 51, 785 (2014).

[17] C. García-Meca and C. Barceló, J. Opt. 18, 044026 (2016).

[18] C. García-Meca, S. Carloni, C. Barceló, G. Jannes, J. SánchezDehesa, and A. Martínez, Sci. Rep. 3, 2009 (2013).
[19] J. C. Halimeh, R. T. Thompson, and M. Wegener, Phys. Rev. A 93, 013850 (2016).

[20] R. Schittny, M. Kadic, T. Bückmann, and M. Wegener, Science 345, 427 (2014).

[21] X. Zhou, G. Hu, and T. Lu, Phys. Rev. B 77, 024101 (2008).

[22] M. D. Guild, A. Alù, and M. R. Haberman, J. Acoust. Soc. Am. 129, 1355 (2011).

[23] R. Schittny, A. Niemeyer, M. Kadic, T. Bückmann, A. Naber, and M. Wegener, Optica 2, 84 (2015).

[24] H. Chen and B. Zheng, Sci. Rep. 2, 255 (2012).

[25] S. Xi, H. Chen, B. I. Wu, and J. A. Kong, IEEE Microw. Compon. Lett. 19, 131 (2009).

[26] N. Landy and D. R. Smith, Nat. Mater. 12, 25 (2012).

[27] A. Bensoussan, G. Papanicolau, and J.-L. Lions, Asymptotic Analysis for Periodic Structures (North-Holland, Amsterdam, 1978).

[28] Y. Huang, Y. Feng, and T. Jiang, Opt. Express 15, 11133 (2007).

[29] C. Qiu, L. Hu, B. Zhang, B.-I. Wu, S. G. Johnson, and J. D. Joannopoulos, Opt. Express 17, 13467 (2009).

[30] R. Schittny, A. Niemeyer, M. Kadic, T. Bückmann, A. Naber, and M. Wegener, Opt. Lett. 40, 4202 (2015).

[31] Y. H. Yang, S. S. Lin, Z. J. Wang, H. Chen, H. Wang, and E. Li, Prog. Electromagn. Res. 142, 31 (2013).

[32] A. Martínez, C. García-Meca, R. Ortuño, F. J. RodríguezFortuño, and J. Martí, Appl. Phys. Lett. 94, 251106 (2009).

[33] R. Schittny, M. Kadic, S. Guenneau, and M. Wegener, Phys. Rev. Lett. 110, 195901 (2013).

[34] T. Han, J. Zhao, T. Yuan, D. Y. Lei, B. Li, and C.-W. Qiu, Energy Environ. Sci. 6, 3537 (2013).

[35] T. Han, X. Bai, D. Gao, J. T. L. Thong, B. Li, and C.-W. Qiu, Phys. Rev. Lett. 112, 054302 (2014).

[36] T. Han, H. Ye, Y. Luo, S. P. Yeo, J. Teng, S. Zhang, and C. W. Qiu, Adv. Mater. 26, 3478 (2014). 